\title{
Patient Reported Outcome (PRO) assessment in epilepsy: a review of epilepsy-specific PROs according to the Food and Drug Administration (FDA) regulatory requirements
}

\author{
Annabel Nixon ${ }^{*}$, Cicely Kerr, Katie Breheny and Diane Wild
}

\begin{abstract}
Despite collection of patient reported outcome (PRO) data in clinical trials of antiepileptic drugs (AEDs), PRO results are not being routinely reported on European Medicines Agency (EMA) and Food and Drug Administration (FDA) product labels. This review aimed to evaluate epilepsy-specific PRO instruments against FDA regulatory standards for supporting label claims. Structured literature searches were conducted in Embase and Medline databases to identify epilepsy-specific PRO instruments. Only instruments that could potentially be impacted by pharmacological treatment, were completed by adults and had evidence of some validation work were selected for review. A total of $26 \mathrm{PROs}$ were reviewed based on criteria developed from the FDA regulatory standards. The ability to meet these criteria was classified as either full, partial or no evidence, whereby partial reflected some evidence but not enough to comprehensively address the FDA regulatory standards. Most instruments provided partial evidence of content validity. Input from clinicians and literature was common although few involved patients in both item generation and cognitive debriefing. Construct validity was predominantly compromised by no evidence of a-priori hypotheses of expected relationships. Evidence for test-retest reliability and internal consistency was available for most PROs although few included complete results regarding all subscales and some failed to reach recommended thresholds. The ability to detect change and interpretation of change were not investigated in most instruments and no PROs had published evidence of a conceptual framework. The study concludes that none of the 26 have the full evidence required by the FDA to support a label claim, and all require further research to support their use as an endpoint. The Subjective Handicap of Epilepsy (SHE) and the Neurological Disorders Depression Inventory for Epilepsy (NDDI-E) have the fewest gaps that would need to be addressed through additional research prior to any FDA regulatory submission, although the NDDI-E was designed as a screening tool and is therefore unlikely to be suitable as an instrument for capturing change in a clinical trial and the SHE lacks the conceptual focus on signs and symptoms favoured by the FDA.
\end{abstract}

Keywords: Patient reported outcomes (PRO), Epilepsy, Review, FDA regulatory requirements

\footnotetext{
* Correspondence: annabel.nixon@oxfordoutcomes.com

Oxford Outcomes, an ICON Plc. Company, Seacourt Tower, West Way, Oxford
} OX2 0JJ, UK 


\section{Introduction}

Epilepsy is defined by the recurrence of spontaneous/unprovoked seizures and covers a range of clinical situations in terms of age of onset, type of seizures, aetiological background, resulting handicap, prognosis, and response to treatment [1]. Epilepsies are a diverse group of disorders with a complex classification, broadly categorised into localization-related, generalized, undetermined, and special syndromes [2]. Antiepileptic drugs (AEDs) are the main treatment option; approximately $60 \%$ of newly diagnosed patients are seizure-free with AED monotherapy and a further $10-20 \%$ with polytherapy [1,3]. Surgery offers alternative treatment options for patients with medically intractable epilepsy [4].

The assessment of efficacy in clinical studies evaluating AEDs is generally focused on seizure frequency/occurrence in line with regulatory guidelines [1]. However there is growing recognition of the value of capturing wider impacts of treatments reported by patients in the form of patient reported outcomes (PROs) [5]. A PRO is defined as any report of the status of a patient's health condition that comes directly from the patient without interpretation of the patient's response by a clinician or anyone else [5]. PROs take the form of carefully designed questionnaires which can be used to capture and quantify the patient experience of treatment and treatment impacts. Given that epilepsy is a complex disorder, which affects patients' psychological health, independence, emotional adjustment and employment [6] there is a strong case for evaluating the impact of AEDs on broader aspects of patient's lives, and previous studies have identified potential areas of patient's lives that may be enhanced if epilepsy symptoms were improved [7]. Therefore it is unsurprising that PROs have been widely incorporated into clinical trials evaluating AEDs [8-10].

In order to guide the selection of PROs for epilepsy clinical studies, there have been numerous reviews evaluating PROs, each review with a unique focus: evaluating PROs designed to measure change in seizure severity [11], overview of neuropsychological and behavioural measures used AED clinical trials [8], guiding health-related quality of life (HRQL) assessment in epilepsy [12], systematically reviewing measures designed to assess the subjective impact of epilepsy and treatment in children and adolescents [13], guiding the selection and use of quality of life (QOL) instruments in epilepsy [9], appraising the conceptual underpinnings of paediatric QOL instruments [14], exploring and reviewing PROs used to assess people with epilepsy [10], and describing QOL instruments for children and adolescents with neurodisabilities [15]. Similar reviews were also conducted prior to $2000[16,17]$.

Despite almost routine collection of PRO data in clinical trials of AEDs and the wide choice of PRO instruments, PRO results are not being routinely reported on European
Medicines Agency (EMA) and Food and Drug Administration (FDA) product labels. A review of the current state of PRO label claims granted for new molecular entities approved 2006-2010 [18] found two approved for the treatment of epilepsy had PRO label claims: Vimpat ${ }^{\circledR}$ indicated as adjunctive therapy for patients $\geq 17$ years to treat partial onset seizures and Sabril ${ }^{\circledR}$ indicated for refractory complex partial seizures in adults. For both products the PRO was a measure of seizure frequency. There are two likely explanations for the lack of PRO label claims for epilepsy treatments. The first relates to the possibility that there may be a lag in the effect of AED treatment on PRO outcomes after demonstration of positive treatment effect on clinical efficacy outcomes e.g. seizure frequency/occurrence. Qualitative studies show that unpredictability of seizures is a key factor in the impact of epilepsy for patients [19-21]. Patients' perception of the unpredictability of their seizures may not change until sometime after a reduction in seizure frequency, or even seizure freedom is achieved; 3-4 months, a common timescale for AED efficacy trials, may be too short to expect much change in PRO endpoints [11]. The second explanation, and the focus of the current review, is the possibility that existing PRO instruments do not meet regulatory requirements.

There is limited guidance from regulatory authorities on incorporating PROs into evaluations of AEDs, the recent EMA guideline on clinical investigation of medicinal products in the treatment of epileptic disorders [1] suggests that a secondary efficacy variable could be 'scales measuring social and working capacity, if validated'. Other than this the authors could find no further guidance from either the EMA or FDA specific to the use of PROs in epilepsy clinical studies. For a PRO to be incorporated into a FDA or EMA label claim, it is necessary for it to be developed to standards required by the regulatory authorities [5,22]. Most of the epilepsy-specific PRO reviews conducted to date were published prior to the current regulatory guidance, and none have evaluated the extent to which PROs have been developed to the standard required by regulatory authorities. In order to guide those developing clinical studies to evaluate AEDs in selecting a PRO instrument for the purpose of obtaining a PRO label claim, this review sought to evaluate existing epilepsy-specific PRO instruments against FDA regulatory standards and identify any gaps in the development process that would need to be addressed prior to regulatory review.

\section{Methods}

\section{Literature search and PRO identification}

Structured Embase and Medline searches were conducted in February 2011 to identify epilepsy studies reporting the use or development of PRO instruments in epilepsy samples. Searches were constructed by combining epilepsy and seizure subject heading search terms with a number 
of PRO measurement terms. Searches were limited to 'humans,' 'English language' and 'year 2000 -current'. Full search terms are shown in the Additional file 1.

Abstracts of publications identified by these searches were screened to identify PRO instruments used. Full text versions of publications were screened to identify specific instruments where abstracts referred to PRO measurement without naming a specific instrument, or where abstracts specified endpoints that may have been captured by PRO measurement. Further electronic searches were conducted for each instrument identified to retrieve a copy of the instrument and establish its relevance. Given the wide variety of PRO instruments used with epilepsy patients, the following instrument inclusion criteria were used to focus the review on broadly comparable instruments that might be used to evaluate pharmacological treatment:

- Epilepsy-specific scale. Based on the FDA's preference for more proximal endpoints that are specifically relevant to the target population, generic PROs which make no reference to epilepsy were excluded.

- Adult patient completed. Most epilepsy-specific PROs have been developed for adult patient completion. Scales developed to assess epilepsy impacts for children are commonly designed for proxy completion (e.g. by parents or clinical assessors) in part or whole and are not directly comparable to PROs designed for adult patient completion. PROs designed for completion by adolescent as well as adult patients were included.

- Target a concept that could potentially be impacted by pharmacological treatment. Non-interventional studies or those evaluating non-pharmacological interventions may also use PROs to evaluate concepts that would not be impacted by pharmacological treatment. However, PROs evaluating these concepts would not need to meet FDA requirements for supporting a label claim.

Further literature searches were then conducted in Embase and Medline to identify published validation work on each PRO instrument identified. Full text versions of all published reviews of PRO instruments identified by the initial search and instrument focused literature searches were retrieved and checked for any additional instruments or validation work. Any instrument that was found to comprise single items that did not form a scale and/or for which no documentary evidence of validation could be located was excluded from further review.

\section{Developing the review criteria}

Detailed review criteria were developed from requirements laid out in the FDA guidance for PROs supporting label claims [5]. These criteria are detailed in Table 1. Development work and evidence for psychometric properties of each PRO instrument were extracted into structured individual instrument review tables before being systematically reviewed against the review criteria (Table 1).

\section{Results}

\section{Identification of PROs}

Initial Embase and Medline searches identified a total of 1854 publications (after de-duplication) from which 159 PRO instruments were identified by abstract or full-text manuscript review. Following instrument retrieval and initial review, 133 of these were excluded as they were found to be not epilepsy specific $(n=52)$, not for adult patient completion $(n=42)$, not to target a concept that could be potentially impacted by pharmacological treatment $(n=20)$ or instruments comprising single items that did not form a scale and/or for which no documentary evidence of validation could be located $(n=19)$. Many of these instruments could be excluded on more than one criterion. Numbers shown here depict the first reason for exclusion.

\section{PRO instrument characteristics}

Twenty-six (26) epilepsy-specific PRO instruments were identified and reviewed, Table 2 details the key characteristics for each of these 26 instruments. The identified PROs vary widely in their key characteristics. Conceptual coverage of the PROs include instruments designed to capture epilepsy attack experience including severity (e.g. Attack Symptom Measure, Ictal Consciousness Inventory (ICI), Liverpool Seizure Severity Scale (LSSS), Seizure Severity Questionnaire (SSQ)); issues associated with the treatment and management of epilepsy (e.g. Assessing Side Effects of AED Treatment (SIDAED), AldenkampBaker Neuroassessment Schedule (ABNAS), Liverpool Adverse Events Profile (LAEP), Portland Neurotoxicity Scale (PNS), Epilepsy Self-Efficacy Scale (ESES)); instruments designed to capture the impacts of epilepsy on different aspects of patient's lives (e.g. Epilepsy Psycho-Social Effects Scale, Impact of Epilepsy Questionnaire, Neurological Disorders Depression Inventory for Epilepsy (NDDI-E), Perceived Stigma Scale, Washington Psychosocial Seizure Inventory (WPSI)) and instruments measuring the impact of epilepsy on patients' HRQL (e.g. Epilepsy Foundation of America (EFA) Concerns Index, Epilepsy Surgery Inventory 55 (ESI-55), EPI-QOL, Quality of Life in Epilepsy (QOLIE) instruments, Quality of Life in Newly Diagnosed Epilepsy (NEWQOL)).

Instruments vary in terms of length, ranging from five items (Perceived Limitations Scale) to 132 items (WPSI). Nine of the PROs do not have a defined recall period (e.g. ABNAS, SIDAED), two refer to the time of an epilepsy attack (Attack Symptom Measure, ICI). Most specify a recall 
Table 1 Review criteria based on FDA requirements for PROs to support label claims

Review criteria

Notation Detail

\begin{tabular}{ll}
$\begin{array}{l}\text { Instrument } \\
\text { property }\end{array}$ & Description from FDA guidance [5] \\
\hline $\begin{array}{l}\text { Conceptual } \\
\text { Framework }\end{array}$ & $\begin{array}{l}\text { An explicit description or diagram of the relationships } \\
\text { between items in a PRO instrument and the concepts } \\
\text { measured, developed from empiric evidence to support } \\
\text { item grouping and scores. }\end{array}$ \\
$\begin{array}{ll}\text { Validity: Content } \\
\text { - Patient Input }\end{array}$ & $\begin{array}{l}\text { Evidence that the instrument measures the concept of } \\
\text { interest including evidence from qualitative studies that } \\
\text { the items and domains of an instrument are appropriate } \\
\text { and comprehensive relative to its intended measurement } \\
\text { concept, population and use. Item generation should } \\
\text { include input from the target population. }\end{array}$ \\
$\begin{array}{l}\text { Validity: Content } \\
\text { - Literature \& }\end{array}$ & $\begin{array}{l}\text { See Validity: Content (above). In addition to focus groups } \\
\text { and individual interviews with patients and family } \\
\text { Clinician Input } \\
\text { members, PRO instrument items can be generated from } \\
\text { literature reviews, interviews with clinicians and other } \\
\text { sources. }\end{array}$
\end{tabular}

\section{$\sqrt{ }$}

$x$

$\checkmark$

Patient involvement in concept elicitation/item generation AND conduct of cognitive debrief with patients.

Partial Some patient involvement in concept elicitation/item generation or cognitive debrief but not both.

$x$

No patient involvement in instrument development and evaluation of content.

Use of literature to guide instrument/item development OR involvement of clinical experts to guide instrument/ item development or evaluate content validity (not necessary to have both).

No use of literature to guide instrument/item development AND no involvement of clinical experts to guide instrument/item development or evaluate content validity.

Validity: Construct Evidence that relationships among items, domains, and concepts conform to a priori hypotheses concerning logical relationships that should exist with other measures (discriminant and convergent validity) or characteristics of patients and patient groups (known groups validity).

Clear hypotheses for relationships with measures (PRO or clinical) of related concepts tested with hypothesised relationships found (convergent validity). Can be supported by evidence that measures of concepts that should not be related show hypothesised lack of
Reliability: Testretest
Stability of scores over time when no change is expected in the concept of interest. correlation (discriminant validity). Can also be supported by known groups validity (hypothesised, tested and found) but known groups validity alone is not sufficient evidence of construct validity.

Partial Construct validity tested without clear hypotheses OR mixed results in terms of the extent to which observed relationships match those hypothesised OR limited number of tests undertaken i.e. if instrument is correlated against one other PRO and that's the extent of the testing, then this is only partial evidence of construct validity.

$\boldsymbol{x} \quad$ Construct validity tested but observed relationships do not match those hypothesised.

Construct validity (convergent, discriminant and known groups validity) not tested.

Correlations $\geq 0.7$ for all scores (including domain scores)

Partial Correlations for some scores $<0.7$ OR good test-retest reliability found for total score but domain scores not evaluated.

$\boldsymbol{x} \quad$ Correlations $<0.7$ for all scores evaluated.

- $\quad$ Not tested for any scores.

Reliability: Internal The extent to which items comprising a scale measure the consistency same concept, intercorrelation of items that contribute to a score.

Cronbach's Alpha $\geq 0.8$ for all scores (including domain scores).

Partial Cronbach's Alpha for some scores $<0.8$ OR good internal consistency found for total score but domain scores not evaluated.

$x$ Cronbach's Alpha $<0.8$ for all scores evaluated.

- $\quad$ Not tested for any scores. 


\section{Table 1 Review criteria based on FDA requirements for PROs to support label claims (Continued)}

\begin{tabular}{ll}
\hline $\begin{array}{l}\text { Ability to detect } \\
\text { change }\end{array}$ & $\begin{array}{l}\text { Evidence that a PRO instrument can identify within person } \\
\text { changes over time in individuals or groups (similar to } \\
\text { those in clinical trials) who are known to have changed } \\
\text { with respect to the measurement concept. }\end{array}$
\end{tabular}

with respect to the measurement concept.
Interpretation of change
The MID is the smallest change in score that can be regarded as important [23]. The FDA guidance uses the term 'responder definition' rather than MID to denote the change in individual PRO score that indicates a treatment benefit. Responder definitions are trial/treatment specific and should be derived empirically using anchor-based methods (clinical anchors or patient ratings of change). Statistically derived responder definitions (e.g. distributionbased methods commonly used to establish MID) can be used to support anchor-based approaches but are not appropriate as the sole basis for determining a responder definition.
Specific aim of analysis was to test within-group responsiveness to change (e.g. set criteria for change e.g. effect sizes), tested and met criteria for all scores (including domain scores). Of key importance is clear evidence/reason to believe that change has occurred in a group (e.g. clinical outcome, anchor-based approach) and that the PRO instrument scores detect this change.

Partial Within-group sensitivity to change criteria met for some but not all scores OR criteria met for a total score but responsiveness of domain scores not tested.

$x$

Within group sensitivity to change tested but criteria not met.

Not tested for any scores. This includes claims of instruments sensitivity to change based on between group change (e.g. difference in change between different arms of clinical trial) and observed change in a group without clear evidence/reason to believe that change has occurred in the group or without the clear aim of evaluating sensitivity to change (e.g. observed change from baseline within one arm of a clinical trial when not evaluated in relation to observed clinical change).

$\checkmark \quad$ Published values for interpretation of change for all scores (including domain scores). Methodological details about how values were derived e.g. statistically, using anchor-based methods, provided and discussed in results text.

Partial Values for interpretation of change for total score but not domain scores. Methodological details about how this was derived e.g. statistically, using anchor-based methods, provided and discussed in results text.

No published evidence for interpretation of change.

\section{Glossary of Terms:}

Cognitive debrief: a qualitative research tool used to determine whether concepts and items are understood by patients in the same way that instrument developers intend.

Concept: the specific measurement goal (i.e. the thing that is to be measured by the PRO instrument).

Item: an individual question, statement or task (and its standardized response options) that is evaluated by the patient to address a particular concept. Reliability: the ability of a PRO instrument to yield consistent, reproducible estimates of true treatment effect.

Responder definition: a score change in a measure, experienced by an individual patient over a predetermined time period that has been demonstrated in the target population to have significant treatment benefit.

period varying from now (EPI-QOL) to six months (SHE), with five PROs referring to multiple recall periods within the same PRO (e.g. EPI-QOL, NEWQOL, SHE). Response options for most of the reviewed PROs employ a Likert approach with 3-5 options, for example the Epilepsy Psycho-Social Effects Scale has a five point Likert scale ranging from 'almost always or always' to 'never' and the EPI-QOL has a five point response scale ranging from 'very frequently' to 'not at all'. Less common is a numerical rating scale (NRS) approach with anchors, such as the ESES which has a 11 point scale ranging from 0 (I cannot do at all) to 10 (sure I can do).

The PROs provide different levels of information depending on the scoring approach, a minority of instruments provide only a total score based on the scoring of all items in the PRO (e.g. EFA Concerns Index, NDDI-E ), whilst most provide a more detailed amount of information through provision of sub-scale scores (also referred to as domain scores), with the number of subscale scores ranging widely, from two (e.g. ICI, PNS) to 20 (Bonner Psychsoziale Skale fur Epilepsie (BPSE)). Most instruments provide between 5-15 subscale scores.

\section{PRO review against regulatory requirements}

Table 3 provides the results of the PRO review against the regulatory requirements detailed in Table 1.

\section{Conceptual framework}

The authors did not find any published conceptual framework for any of the reviewed instruments.

\section{Content validity}

Three of the PROs involved patients in concept elicitation/item generation and in the evaluation of items through a cognitive debrief methodology or similar 


\section{Table 2 Epilepsy-specific PRO instruments}

\begin{tabular}{|c|c|c|c|c|c|c|c|}
\hline $\begin{array}{l}\text { Abbreviated } \\
\text { name }\end{array}$ & Full name & Overview & $\begin{array}{l}\text { Scoring: total score \& sub- } \\
\text { scales }\end{array}$ & $\begin{array}{l}\text { Number of } \\
\text { items }\end{array}$ & $\begin{array}{l}\text { Recall } \\
\text { period }\end{array}$ & Response options & $\begin{array}{l}\text { Published } \\
\text { details of } \\
\text { instrument } \\
\text { development } \\
\text { and } \\
\text { psychometric } \\
\text { properties }\end{array}$ \\
\hline \multirow[t]{13}{*}{ ABNAS } & \multirow{7}{*}{$\begin{array}{l}\text { Aldenkamp-Baker } \\
\text { Neuroassessment } \\
\text { Schedule }\end{array}$} & \multirow{7}{*}{$\begin{array}{l}\text { An instrument to measure patient } \\
\text { perceived cognitive side effects of AED } \\
\text { treatment. }\end{array}$} & $\begin{array}{l}\text { Total score and } 6 \text { subscale } \\
\text { scores: }\end{array}$ & \multirow[t]{7}{*}{24} & \multirow[t]{7}{*}{$\begin{array}{l}\text { No recall } \\
\text { period }\end{array}$} & \multirow[t]{7}{*}{$\begin{array}{l}\text { Four point scale ranging from 'no } \\
\text { problem' to 'a serious problem' }\end{array}$} & \multirow[t]{7}{*}[24-28]{} \\
\hline & & & 1. Fatigue & & & & \\
\hline & & & 2. Slowing & & & & \\
\hline & & & 3. Memory & & & & \\
\hline & & & 4. Concentration & & & & \\
\hline & & & 5. Language & & & & \\
\hline & & & 6. Motor coordination & & & & \\
\hline & \multirow[t]{6}{*}{$\begin{array}{l}\text { Attack Symptom } \\
\text { Measure }\end{array}$} & \multirow{6}{*}{$\begin{array}{l}\text { An instrument to measure ictal } \\
\text { symptoms that would typically be } \\
\text { associated with panic disorder. }\end{array}$} & $\begin{array}{l}\text { Total score and five sub- } \\
\text { scales: }\end{array}$ & \multirow[t]{6}{*}{26} & \multirow[t]{6}{*}{$\begin{array}{l}\text { During their } \\
\text { attacks }\end{array}$} & \multirow[t]{6}{*}{$\begin{array}{l}\text { Two options indicating the absence or } \\
\text { presence of the symptom }\end{array}$} & \multirow[t]{6}{*}{ [29] } \\
\hline & & & $\begin{array}{l}\text { 1. Autonomic arousal } \\
\text { symptoms }\end{array}$ & & & & \\
\hline & & & $\begin{array}{l}\text { 2. Chest and abdomen } \\
\text { symptoms }\end{array}$ & & & & \\
\hline & & & 3. Mental state symptoms & & & & \\
\hline & & & 4. General symptoms & & & & \\
\hline & & & 5. Cognitive symptoms & & & & \\
\hline \multirow[t]{13}{*}{ BPSE } & \multirow{13}{*}{$\begin{array}{l}\text { Bonner } \\
\text { Psychsoziale } \\
\text { Skalen fur } \\
\text { Epilepsie }\end{array}$} & \multirow{13}{*}{$\begin{array}{l}\text { A self-report questionnaire designed to } \\
\text { evaluate the patient's areas of illness- } \\
\text { related psychosocial problems and } \\
\text { cognitive-behavioural variables. }\end{array}$} & 20 subscales in six areas: & \multirow[t]{13}{*}{87} & \multirow{13}{*}{$\begin{array}{l}\text { No recall } \\
\text { period in } \\
\text { example } \\
\text { items } \\
\text { reviewed }\end{array}$} & \multirow{13}{*}{$\begin{array}{l}\text { Analogue ratings of between } 0 \text { to } 6 \text {. } \\
\text { Scales include Never to Always or Not } \\
\text { at all to Extremely and primarily focus } \\
\text { on frequency of cognitions or } \\
\text { behaviour }\end{array}$} & \multirow[t]{13}{*}[30]{} \\
\hline & & & 1. Impairment to daily life & & & & \\
\hline & & & a. Physically & & & & \\
\hline & & & b. Activity/capability & & & & \\
\hline & & & c. Relations and family & & & & \\
\hline & & & d. Emotional/mood & & & & \\
\hline & & & e. Independence & & & & \\
\hline & & & f. Problem-solving/coping & & & & \\
\hline & & & 2. Environmental experiences & & & & \\
\hline & & & a. Positive & & & & \\
\hline & & & b .Negative & & & & \\
\hline & & & c. Present emotions/moods & & & & \\
\hline & & & 3. Attitude to illness/seizures & & & & \\
\hline
\end{tabular}




\begin{tabular}{|c|c|c|c|c|c|c|c|}
\hline & & & a. Helplessess & & & & \\
\hline & & & b. External attribution & & & & \\
\hline & & & c. Internal attribution & & & & \\
\hline & & & $\begin{array}{l}\text { 4. Relatives' reaction to } \\
\text { seizures }\end{array}$ & & & & \\
\hline & & & a. Calming down/relief & & & & \\
\hline & & & b. Punishment & & & & \\
\hline & & & c. Distraction & & & & \\
\hline & & & 5. Activities & & & & \\
\hline & & & a. Home/family & & & & \\
\hline & & & b. Social & & & & \\
\hline & & & c. Cultural & & & & \\
\hline & & & $\begin{array}{l}\text { 6. Influences on seizure } \\
\text { occurrence }\end{array}$ & & & & \\
\hline & & & a. Personal/others & & & & \\
\hline $\begin{array}{l}\text { EFA concerns } \\
\text { index }\end{array}$ & $\begin{array}{l}\text { Epilepsy } \\
\text { Foundation of } \\
\text { America concerns } \\
\text { index }\end{array}$ & $\begin{array}{l}\text { A HRQL measure for patients with } \\
\text { epilepsy, covering concerns relating to } \\
\text { affective enjoyment, general autonomy, } \\
\text { seizure recurrence, family burden and } \\
\text { lack of understanding. }\end{array}$ & $\begin{array}{l}\text { Ratings are summed to } \\
\text { produce a Concerns Index } \\
\text { Score. }\end{array}$ & 20 & Past 4 weeks & $\begin{array}{l}\text { Five point scales ranging from 'not at all } \\
\text { concerned' to 'extremely concerned' or } \\
\text { 'none of the time' to 'all of the time' }\end{array}$ & {$[31-33]$} \\
\hline \multirow[t]{8}{*}{ EPSES } & \multirow{8}{*}{$\begin{array}{l}\text { Epilepsy Psycho- } \\
\text { Social Effects } \\
\text { Scale }\end{array}$} & \multirow{8}{*}{$\begin{array}{l}\text { An instrument to determine the social } \\
\text { effects of epilepsy and measure } \\
\text { psychosocial functioning. }\end{array}$} & $\begin{array}{l}\text { Total weighted score and } 14 \\
\text { subscale scores: }\end{array}$ & \multirow[t]{8}{*}{42} & \multirow[t]{8}{*}{$\begin{array}{l}\text { No recall } \\
\text { period }\end{array}$} & \multirow[t]{8}{*}{$\begin{array}{l}\text { Five point scale ranging from 'almost } \\
\text { always or always' to 'never' }\end{array}$} & \multirow[t]{8}{*}[34]{} \\
\hline & & & $\begin{array}{l}\text { 1. Attitude towards accepting } \\
\text { attacks }\end{array}$ & & & & \\
\hline & & & 2. Fear of having seizures & & & & \\
\hline & & & $\begin{array}{l}\text { 3. Fear of stigma in } \\
\text { employment }\end{array}$ & & & & \\
\hline & & & $\begin{array}{l}\text { 4. Lack of confidence about } \\
\text { the future }\end{array}$ & & & & \\
\hline & & & $\begin{array}{l}\text { 5. Lack of confidence about } \\
\text { travelling }\end{array}$ & & & & \\
\hline & & & $\begin{array}{l}\text { 6. Adverse reaction on social } \\
\text { life }\end{array}$ & & & & \\
\hline & & & $\begin{array}{l}\text { 8. Change in outlook on life/ } \\
\text { self }\end{array}$ & & & & \\
\hline
\end{tabular}




\begin{tabular}{|c|c|c|c|c|c|c|c|}
\hline & & & $\begin{array}{l}\text { 9. Difficulty communicating } \\
\text { with the family }\end{array}$ & & & & \\
\hline & & & $\begin{array}{l}\text { 10. Problems with taking } \\
\text { medication }\end{array}$ & & & & \\
\hline & & & $\begin{array}{l}\text { 11. Distrust of the medical } \\
\text { profession }\end{array}$ & & & & \\
\hline & & & $\begin{array}{l}\text { 12. Depression or emotional } \\
\text { reactions }\end{array}$ & & & & \\
\hline & & & $\begin{array}{l}\text { 13. Feeling of increased socia } \\
\text { isolation }\end{array}$ & & & & \\
\hline & & & 14. Lethargy/lack of energy & & & & \\
\hline \multirow[t]{7}{*}{ - } & \multirow[t]{7}{*}{ EPI-QOL } & \multirow[t]{7}{*}{$\begin{array}{l}\text { A HRQL assessment instrument for } \\
\text { adults with epilepsy. }\end{array}$} & $\begin{array}{l}\text { Total score and six subscale } \\
\text { scores: }\end{array}$ & 46 & \multirow{7}{*}{$\begin{array}{l}\text { Past two } \\
\text { weeks and } \\
\text { now }\end{array}$} & \multirow[t]{7}{*}{$\begin{array}{l}\text { Five point scale ranging from 'very } \\
\text { frequently' to 'not at all'. }\end{array}$} & \multirow[t]{7}{*}{ [35] } \\
\hline & & & 1. Physical functioning & & & & \\
\hline & & & 2. Emotional wellbeing & & & & \\
\hline & & & 3. Cognitive functioning & & & & \\
\hline & & & 4. Social functioning & & & & \\
\hline & & & 5. Seizure worry & & & & \\
\hline & & & 6. Medication effects & & & & \\
\hline ESES & $\begin{array}{l}\text { Epilepsy Self- } \\
\text { Efficacy Scale }\end{array}$ & $\begin{array}{l}\text { An instrument to measure self-efficacy } \\
\text { in regards to epilepsy management. }\end{array}$ & Total score. & 33 & $\begin{array}{l}\text { No recall } \\
\text { period }\end{array}$ & $\begin{array}{l}\text { Ten point scale ranging from 'I cannot } \\
\text { do at all' to 'Sure I can do' }\end{array}$ & {$[36,37]$} \\
\hline \multirow[t]{11}{*}{ ESI-55 } & \multirow[t]{11}{*}{$\begin{array}{l}\text { Epilepsy Surgery } \\
\text { Inventory } 55\end{array}$} & \multirow[t]{11}{*}{$\begin{array}{l}\text { An instrument to measure HRQL in } \\
\text { epilepsy surgery patients. }\end{array}$} & $\begin{array}{l}\text { Weighted total score, three } \\
\text { composite scores (Physical, } \\
\text { mental and cognitive role) } \\
\text { and eleven subscale scores: }\end{array}$ & 55 & \multirow[t]{11}{*}{$\begin{array}{l}\text { Various } \\
\text { today/last } \\
\text { week/last } \\
\text { year/4 weeks }\end{array}$} & \multirow[t]{11}{*}{$\begin{array}{l}\text { Various response formats. ('strongly } \\
\text { agree' to strongly disagree', 'often' to } \\
\text { 'not at all', yes/no) }\end{array}$} & \multirow[t]{11}{*}[38,39]{} \\
\hline & & & 1. Emotional wellbeing & & & & \\
\hline & & & $\begin{array}{l}\text { 2. Role limitations due to } \\
\text { emotional problems }\end{array}$ & & & & \\
\hline & & & 3. Energy/fatigue & & & & \\
\hline & & & 4. Social function & & & & \\
\hline & & & 5. Pain & & & & \\
\hline & & & 6. Physical function & & & & \\
\hline & & & $\begin{array}{l}\text { 7. Role limitations due to } \\
\text { physical problems }\end{array}$ & & & & \\
\hline & & & 8. Health perceptions & & & & \\
\hline & & & 9. Cognitive function & & & & \\
\hline & & & $\begin{array}{l}\text { 10. Role limitations due to } \\
\text { memory problems }\end{array}$ & & & & \\
\hline
\end{tabular}


Table 2 Epilepsy-specific PRO instruments (Continued)

11.Overall quality of life

\begin{tabular}{|c|c|c|c|c|c|c|c|}
\hline \multirow[t]{3}{*}{$\mathrm{ICl}$} & \multirow{3}{*}{$\begin{array}{l}\text { Ictal } \\
\text { Consciousness } \\
\text { Inventory }\end{array}$} & \multirow{3}{*}{$\begin{array}{l}\text { A quantitative assessment of the level of } \\
\text { awareness and content of ictal } \\
\text { consciousness. }\end{array}$} & Two sub-scale scores: & \multirow[t]{3}{*}{20} & \multirow{3}{*}{$\begin{array}{l}\text { During a } \\
\text { single seizure }\end{array}$} & \multirow{3}{*}{$\begin{array}{l}\text { Three point scale: 'no'; 'yes, a bit (yes, } \\
\text { vaguely)'; 'yes, much (yes, clearly)'. }\end{array}$} & \multirow[t]{3}{*}[40]{} \\
\hline & & & $\begin{array}{l}\text { 1. Level of consciousness } \\
\text { (ICl-L) }\end{array}$ & & & & \\
\hline & & & $\begin{array}{l}\text { 2. Content of consciousness } \\
\text { (ICl-C) }\end{array}$ & & & & \\
\hline IES & $\begin{array}{l}\text { Impact of } \\
\text { Epilepsy Scale }\end{array}$ & $\begin{array}{l}\text { Developed to assess the impact of } \\
\text { epilepsy and antiepileptic drug therapy } \\
\text { on an individual's relationship with } \\
\text { friends and family, social life, } \\
\text { employment, health, self-esteem, plans } \\
\text { for the future, and standard of living. }\end{array}$ & Total impact score. & 8 & $\begin{array}{l}\text { No recall } \\
\text { period. }\end{array}$ & $\begin{array}{l}\text { Four point scale ranging from 'not at all' } \\
\text { to 'a lot' }\end{array}$ & {$[41,42]$} \\
\hline \multirow[t]{2}{*}{ LAEP } & \multirow{2}{*}{$\begin{array}{l}\text { Liverpool Adverse } \\
\text { Events Profile }\end{array}$} & \multirow[b]{2}{*}{$\begin{array}{l}\text { An instrument to measure total side } \\
\text { effects burden of a medical regimen. It } \\
\text { was developed to evaluate the most } \\
\text { common negative side effects reported } \\
\text { by patients taking AEDs. The AEP } \\
\text { evaluates the interiactal state. It's widely } \\
\text { used alone and is also part of the } \\
\text { Liverpool Battery. }\end{array}$} & \multirow[t]{2}{*}{ Total score. } & 19 & \multirow[t]{2}{*}{ Past 4 weeks } & \multirow{2}{*}{$\begin{array}{l}\text { Four point scale ranging from 'never a } \\
\text { problem' to 'always or often a problem' }\end{array}$} & \multirow[t]{2}{*}{ [43-49] } \\
\hline & & & & $\begin{array}{l}\text { Space to rate } \\
\text { up to } 3 \\
\text { additional } \\
\text { AEs. }\end{array}$ & & & \\
\hline LSSS & $\begin{array}{l}\text { Liverpool Seizure } \\
\text { Severity Scale } 2.0 \\
\text { (2001) }\end{array}$ & $\begin{array}{l}\text { A scale designed to quantify patient's } \\
\text { own perceptions of seizure severity. }\end{array}$ & $\begin{array}{l}\text { Weighted most severe ictal } \\
\text { effects score. }\end{array}$ & 12 & Past 4 weeks & $\begin{array}{l}\text { Various scales referring to the content } \\
\text { of each question ranging from } 4 \text { to } 6 \\
\text { options. For example 'I always feel } \\
\text { sleepy' to 'I never feel sleepy' }\end{array}$ & {$[17,44,50-53]$} \\
\hline NDDI-E & $\begin{array}{l}\text { Neurological } \\
\text { Disorders } \\
\text { Depression } \\
\text { Inventory for } \\
\text { Epilepsy }\end{array}$ & $\begin{array}{l}\text { An instrument to detect depression in } \\
\text { epilepsy patients. }\end{array}$ & Total score. & 6 & $\begin{array}{l}\text { Past two } \\
\text { weeks }\end{array}$ & $\begin{array}{l}\text { Four point frequency scale ranging from } \\
\text { 'always or often' to 'never'. }\end{array}$ & [54] \\
\hline \multirow[t]{9}{*}{ NEWQOL } & \multirow{9}{*}{$\begin{array}{l}\text { Quality of Life in } \\
\text { Newly Diagnosed } \\
\text { Epilepsy }\end{array}$} & \multirow{9}{*}{$\begin{array}{l}\text { An instrument to measure aspects of } \\
\text { quality of life postulated as being } \\
\text { important for patients recently } \\
\text { diagnosed with epilepsy. }\end{array}$} & $\begin{array}{l}\text { Eight subscale scores and } \\
\text { single item scores: }\end{array}$ & 93 & \multirow{9}{*}{$\begin{array}{l}\text { Varies from } \\
\text { 'How you are } \\
\text { now' to 'In } \\
\text { the last year' }\end{array}$} & \multirow{9}{*}{$\begin{array}{l}\text { Various response formats including } 4 \\
\text { and } 5 \text { point scales ranging from 'no } \\
\text { problem' to 'a serious problem' or } \\
\text { 'severely restricted' to 'not restricted at } \\
\text { all' }\end{array}$} & \multirow[t]{9}{*}[25]{} \\
\hline & & & 1. anxiety & & & & \\
\hline & & & 2. depression & & & & \\
\hline & & & 3. social activities, & & & & \\
\hline & & & 4. symptoms, & & & & \\
\hline & & & 5. locus of control/mastery, & & & & \\
\hline & & & $\begin{array}{l}\text { 6. neuropsychological } \\
\text { problems (subscales of } \\
\text { fatigue, memory, } \\
\text { concentration, motor skills, } \\
\text { reading), }\end{array}$ & & & & \\
\hline & & & 7. social stigma*, worry & & & & \\
\hline & & & 8. work limitations. & & & & \\
\hline
\end{tabular}




\begin{tabular}{|c|c|c|c|c|c|c|c|}
\hline & & & Several single-item measures: & & & & \\
\hline & & & •general health & & & & \\
\hline & & & •number of seizures & & & & \\
\hline & & & -social limitations & & & & \\
\hline & & & -social support & & & & \\
\hline & & & •ambition & & & & \\
\hline & & & -limitations & & & & \\
\hline & & & ·health transition & & & & \\
\hline & & & -general limitations & & & & \\
\hline & $\begin{array}{l}\text { Perceived } \\
\text { Limitations Scale }\end{array}$ & $\begin{array}{l}\text { A measure of the constraints that } \\
\text { patients with epilepsy might experience } \\
\text { including the sense of vulnerability to } \\
\text { the physical consequences of seizures. }\end{array}$ & Total score. & 5 & $\begin{array}{l}\text { No recall } \\
\text { period }\end{array}$ & $\begin{array}{l}\text { Four point scale ranging from 'strongly } \\
\text { agree' to 'strongly disagree' }\end{array}$ & {$[55]$} \\
\hline & $\begin{array}{l}\text { Perceived Stigma } \\
\text { Scale }\end{array}$ & $\begin{array}{l}\text { A measure of the extent to which } \\
\text { people with epilepsy feel they are } \\
\text { victims to prejudice, including the } \\
\text { extent to which individuals are treated } \\
\text { differently and inability to change the } \\
\text { views of others. }\end{array}$ & Total score. & 6 & $\begin{array}{l}\text { No recall } \\
\text { period }\end{array}$ & $\begin{array}{l}\text { Four point scale ranging from 'strongly } \\
\text { agree' to 'strongly disagree' }\end{array}$ & {$[55]$} \\
\hline \multirow[t]{8}{*}{ PESOS } & \multirow{8}{*}{$\begin{array}{l}\text { Performance, } \\
\text { subjective } \\
\text { evaluation and } \\
\text { socio- } \\
\text { demographic } \\
\text { data }\end{array}$} & \multirow{8}{*}{$\begin{array}{l}\text { A test-battery for assessing the severity } \\
\text { of epilepsy, epilepsy related quality of } \\
\text { life, restrictions in daily life and } \\
\text { psychosocial problems. }\end{array}$} & $\begin{array}{l}\text { Separate scores for each } \\
\text { instrument in the battery: }\end{array}$ & \multirow[t]{8}{*}{69} & \multirow[t]{8}{*}{$\begin{array}{l}\text { Various recall } \\
\text { periods }\end{array}$} & \multirow[t]{8}{*}{ Various response options } & \multirow[t]{8}{*}[56]{} \\
\hline & & & 1. Restrictions in daily life $\wedge$ & & & & \\
\hline & & & 2. Epilepsy related fear ${ }^{5}$ & & & & \\
\hline & & & 3. Stigma & & & & \\
\hline & & & 4. Emotional adaptation & & & & \\
\hline & & & 5. Problems at work & & & & \\
\hline & & & 6. Problems at school & & & & \\
\hline & & & 7. Problems with parents & & & & \\
\hline \multirow[t]{3}{*}{ PNS } & \multirow{3}{*}{$\begin{array}{l}\text { Portland } \\
\text { Neurotoxicity } \\
\text { Scale }\end{array}$} & \multirow{3}{*}{$\begin{array}{l}\text { An instrument to measure commonly } \\
\text { experienced adverse events associated } \\
\text { with AEDs. }\end{array}$} & $\begin{array}{l}\text { Total score and two subscale } \\
\text { scores: }\end{array}$ & \multirow[t]{3}{*}{15} & \multirow[t]{3}{*}{$\begin{array}{l}\text { Past few } \\
\text { weeks }\end{array}$} & \multirow[t]{3}{*}{$\begin{array}{l}\text { Nine options ranging from 'no problem' } \\
\text { to 'severe problem' }\end{array}$} & \multirow[t]{3}{*}{ [57] } \\
\hline & & & 1. Cognitive toxicity & & & & \\
\hline & & & 2. Somatomotor toxicity & & & & \\
\hline
\end{tabular}


Table 2 Epilepsy-specific PRO instruments (Continued)

\begin{tabular}{|c|c|c|c|c|c|c|c|}
\hline \multirow[t]{5}{*}{ QOLIE-10 } & \multirow[t]{5}{*}{$\begin{array}{l}\text { Quality of life in } \\
\text { epilepsy } 10\end{array}$} & \multirow[t]{5}{*}{$\begin{array}{l}\text { A brief measure of overall quality of life } \\
\text { for patients with epilepsy. }\end{array}$} & $\begin{array}{l}\text { Total score and three } \\
\text { subscale scores: }\end{array}$ & \multirow[t]{5}{*}{10} & \multirow[t]{5}{*}{ Past 4 weeks } & \multirow{5}{*}{$\begin{array}{l}\text { Various } 5 \text { point scales including 'not at } \\
\text { all'to 'a great deal' and 'not at all fearful' } \\
\text { to 'extremely fearful' }\end{array}$} & \multirow[t]{5}{*}[58,59]{} \\
\hline & & & 1. Epilepsy effects & & & & \\
\hline & & & 2. Mental health & & & & \\
\hline & & & 3. Role functioning & & & & \\
\hline & & & $\begin{array}{l}\text { Alternatively scores are } \\
\text { calculated for the } 7 \text { domains } \\
\text { of the QOLIE-31, although for } \\
\text { the QOLIE-10, } 5 \text { of the } 7 \\
\text { subscales are scored from } \\
\text { single items. }\end{array}$ & & & & \\
\hline \multirow[t]{8}{*}{ QOLIE-31 } & \multirow[t]{8}{*}{$\begin{array}{l}\text { Quality of life in } \\
\text { epilepsy } 31\end{array}$} & \multirow[t]{8}{*}{$\begin{array}{l}\text { Overall quality of life for patients with } \\
\text { epilepsy. }\end{array}$} & $\begin{array}{l}\text { Total weighted score and } \\
\text { seven subscale scores: }\end{array}$ & \multirow[t]{8}{*}{31} & \multirow[t]{8}{*}{ Past 4 weeks } & \multirow{8}{*}{$\begin{array}{l}\text { Various response formats including five } \\
\text { and six point scales ranging from 'very } \\
\text { fearful' to 'not fearful at all' or all the } \\
\text { time' to 'none of the time' }\end{array}$} & \multirow[t]{8}{*}[44,59-63]{} \\
\hline & & & 1. Seizure worry & & & & \\
\hline & & & 2. Overall quality of life & & & & \\
\hline & & & 3. Emotional well-being & & & & \\
\hline & & & 4. Energy/fatigue & & & & \\
\hline & & & 5. Cognitive functioning & & & & \\
\hline & & & 6. Medication effects & & & & \\
\hline & & & 7. Social functioning & & & & \\
\hline \multirow[t]{15}{*}{ QOLIE-89 } & \multirow[t]{15}{*}{$\begin{array}{l}\text { Quality of life in } \\
\text { epilepsy } 89\end{array}$} & \multirow[t]{15}{*}{$\begin{array}{l}\text { Overall quality of life for patients with } \\
\text { epilepsy }\end{array}$} & $\begin{array}{l}\text { Total weighted score and } 17 \\
\text { subscale scores: }\end{array}$ & \multirow[t]{15}{*}{89} & \multirow[t]{15}{*}{ Past 4 weeks } & \multirow{15}{*}{$\begin{array}{l}\text { Various response formats including yes/ } \\
\text { no or } 5 \text { point scales ranging from } \\
\text { 'excellent' to 'poor' or 'all the time' to } \\
\text { 'none of the time' }\end{array}$} & \multirow[t]{15}{*}[44,60-67]{} \\
\hline & & & 1. Seizure worry & & & & \\
\hline & & & 2. medication effects & & & & \\
\hline & & & 3. health perceptions & & & & \\
\hline & & & 4. health discouragement & & & & \\
\hline & & & 5. work/driving/social function & & & & \\
\hline & & & 6. language & & & & \\
\hline & & & 7. attention/concentration & & & & \\
\hline & & & 8. memory & & & & \\
\hline & & & 9. overall QOL & & & & \\
\hline & & & 10. emotional wellbeing & & & & \\
\hline & & & 11. role limitations: emotional & & & & \\
\hline & & & 12. role limitations: physical & & & & \\
\hline & & & 13. social isolation, & & & & \\
\hline & & & 14. social support & & & & \\
\hline
\end{tabular}


Table 2 Epilepsy-specific PRO instruments (Continued)

15. energy/fatigue

16. physical functioning

17. pain.

SEALS

\section{Side Effect and} Life Satisfaction Inventory

A self-report questionnaire designed to measure satisfaction with AED therapy.

.

Total score and five subscale scores:

1. Cognition

2. Dysphoria

3. Tiredness

4. Temper

5. Worry

\section{Subjective} Handicap of Epilepsy

\begin{abstract}
An instrument to assess the duration and severity of adverse events that are possibly AED related.

Measures patient's subjective handicap of epilepsy based on the WHO concept

of handicap. It is recommended for use

in studying the long-term consequences

of medical, psychosocial and
interventions in epilepsy.

interventions in epilepsy.
\end{abstract}

Six subscale scores:
1. Work and activities

2. Social and personal

3. Physical

4. Self-perception

5. Life satisfaction

6. Change

Total score.

Adverse events can also be grouped in 10 categories

1. General CNS

2. Behaviour (increased irritability)

3. Depressive symptoms

4. Cognitive function

5. Motor problems and coordination

6. Visual complaints

7. Headache

8. Cosmetic and

dermatological complaints

9. Gastrointestinal complaints

10. Sexuality and menses
Past week Four point scale ranging from 'never' to [68-71] 'many times'.

$\begin{array}{ll}32 & \text { Past } \\ \text { Some } & 6 \text { months, } \\ \text { responses } & \text { except for } \\ \text { are optional } & \text { the change } \\ \text { based upon } & \text { scale which } \\ \text { the patient's } & \text { is the last } \\ \text { situation. } & \text { year }\end{array}$

Various 5 point scales. For example, 'Much better' to 'Much worse' or 'Very often' to 'never'. 
Table 2 Epilepsy-specific PRO instruments (Continued)

\begin{tabular}{|c|c|c|c|c|c|c|c|}
\hline \multirow[t]{4}{*}{ SSQ } & \multirow{4}{*}{$\begin{array}{l}\text { Seizure Severity } \\
\text { Questionnaire }\end{array}$} & \multirow{4}{*}{$\begin{array}{l}\text { An instrument designed to assess } \\
\text { seizure severity as a treatment response. } \\
\text { The measure asks about events before, } \\
\text { during and after a seizure and covers } \\
\text { bother, severity and frequency of } \\
\text { seizures. }\end{array}$} & Three subscale scores: & \multirow[t]{4}{*}{22} & \multirow[t]{4}{*}{ Past 4 weeks } & \multirow{4}{*}{$\begin{array}{l}\text { Various response formats. Format is } \\
\text { primarily seven point scales referring to } \\
\text { frequency, ranging from 'never' to } \\
\text { 'always', bother, ranging from 'no } \\
\text { bother at all' to 'very bothersome' and } \\
\text { severity 'very mild' to 'very severe', } \\
\text { There are also yes/no responses. }\end{array}$} & \multirow[t]{4}{*}{ [78] } \\
\hline & & & 1. Seizure severity & & & & \\
\hline & & & 2. Overall assessment & & & & \\
\hline & & & $\begin{array}{l}\text { 3. Change after alteration of } \\
\text { treatment }\end{array}$ & & & & \\
\hline \multirow[t]{9}{*}{ WPSI } & \multirow{9}{*}{$\begin{array}{l}\text { Washington } \\
\text { Psychosocial } \\
\text { Seizure Inventory }\end{array}$} & \multirow{9}{*}{$\begin{array}{l}\text { Provides absolute and relative estimates } \\
\text { of psychosocial functioning. }\end{array}$} & Eight subscale scores: & \multirow[t]{9}{*}{132} & \multirow{9}{*}{$\begin{array}{l}\text { No recall } \\
\text { period }\end{array}$} & \multirow{9}{*}{$\begin{array}{l}\text { Yes/no responses reflecting self- } \\
\text { perceived feelings and actions. }\end{array}$} & \multirow[t]{9}{*}{ [79-81] } \\
\hline & & & 1.Family background & & & & \\
\hline & & & 2. emotional adjustment & & & & \\
\hline & & & 3. interpersonal adjustment & & & & \\
\hline & & & 4. financial status & & & & \\
\hline & & & 5. adjustment to seizures & & & & \\
\hline & & & 6. medicine & & & & \\
\hline & & & 7. medicinal management & & & & \\
\hline & & & $\begin{array}{l}\text { 8. overall psychosocial } \\
\text { functioning }\end{array}$ & & & & \\
\hline
\end{tabular}

*Stigma of epilepsy scale has been used as a stand-alone PRO instrument to measure epilepsy patient's perception of stigma. It comprises 3 items forming a total score [81-84].

$\wedge$ Restrictions in daily life has been used as a stand-alone PRO instrument to measure social, physical and psychological dimensions that are generally stressed as central aspects of HRQL. It comprises 11 items representing three sub-scales: independent living and mobility, physical and emotional health, partnership, family and friends [56].

$\$$ Epilepsy Related Fears has been used as a stand-alone PRO instrument to measure fears regarding aspects of physical and social consequences. Comprising 11 items representing two sub-scales: Physical Consequences, Social Consequences [56].

AED: anti-epileptic drug. 
approach: the EFA, LSSS and SHE. Of these, the LSSS and SHE also had documented evidence that literature had been used to guide the instrument development and/or clinical experts were involved. Most of the PROs ( $\mathrm{n}=14$ ) had partially involved patients in the development of the instrument, in most cases either concept elicitation OR cognitive debrief were undertaken, but not both methodologies. All but one of these 14 instruments (LAEP) had also reviewed literature and/or involved clinical experts in the instrument development. Of the 26 instruments only five did not involve literature and/or clinical experts in the instrument development process according to published information.

\section{Construct validity}

Seven instruments had full evidence of the construct validity of the PRO by providing hypotheses of the expected relationships between the PRO under evaluation and other clinical or PRO measures, and the hypotheses being supported by reported results. Most of the PROs only had partial evidence of construct validity $(\mathrm{n}=16)$, in most cases there was no evidence of hypotheses of expected relationships being developed in advance of analysis. For some instruments this was compounded by limited testing e.g. only known groups validity was evaluated or very limited comparisons were made. The Perceived Limitations Scale, Perceived Stigma Scale and SIDAED did not have any available evidence of construct validity.

\section{Reliability}

Five PROs had sufficient evidence of test-retest reliability, and four PROs had sufficient evidence of internal consistency reliability, with two PROs providing sufficient evidence of both types of reliability: LAEP and NDDI-E. Thirteen PROs had partial evidence of test-retest reliability and 14 had partial evidence of internal consistency. Most of the PROs that had only partial evidence of these measurement properties had a mix of results for subscales in terms of achieving the required criteria (i.e. $\alpha \geq$ 0.8 for internal consistency $[85,86], r \geq 0.7$ for test-retest reliability) (e.g. PESOS, EPI-QOL) or there were no results provided for the PROs total score (e.g. Attack Symptom Measure). Eight PROs had no evidence of test-retest reliability (Attack Symptoms Measure, BPSE, ESI-55, ICI, Impact of Epilepsy scale (IES), Perceived Limitations Scale, Perceived Stigma Scale and SIDEAD) and four instruments had no evidence of internal consistency reliability (Epilepsy Psycho-Social Effects Scale, Portland Neurotoxicity Scale, SIDAED and SSQ).

Four PROs had evidence of testing for internal consistency but failed to reach the required standard of $\alpha \geq 0.8$ for all reported scales including any total score: Quality of Life in Epilepsy 10 (QOLIE 10), Perceived
Stigma Scale, Perceived Limitations Scale and IES. The IES has an internal consistency of 0.65 which increases to 0.82 if one of the 8 items is removed, but later publications are based on either the eight item version or a 10 -item version $[42,87]$ for which no published psychometric validation evidence could be found. The Perceived Limitations Scale had a notably low internal consistency $(\alpha=0.55)$ with the Perceived Stigma Scale getting close to the required standard $(\alpha=0.75)$ [55]. The reported alpha values for the three empirically derived factors from the QOLIE-10 (epilepsy effects, mental health scale, role function) do not meet the criterion thresholds [58]. These three subscales are not the usual scores derived from the QOLIE-10. Researchers more commonly report the same seven subscales as the QOLIE-31 (five of which have only one item in the QOLIE-10) and/or a total QOLIE-10 score $[59,88,89]$ for which no evaluation of internal consistency has been published.

\section{Ability to detect change}

Four PROs had full evidence of ability to detect change and reported the results of analysis undertaken with the specific aim of testing within-group responsiveness for all sub-scales as well as total score (as appropriate): ESI55, Performance, subjective evaluation and sociodemographic data (PESOS), Quality of Life in Epilepsy 31 (QOLIE-31) and Quality of Life in Epilepsy 89 (QOLIE-89). Three PROs provided partial evidence of ability to detect change: LSSS, QOLIE-10 and WPSI; for example analysis was conducted on a previous version of the PRO (LSSS) or a non-empirically derived scale structure (QOLIE-10 results based on sub-scales taken from the QOLIE-31). For most of the PROs $(n=19)$ this measurement property had not been investigated.

\section{Interpretation of change}

The LAEP was the only PRO that had fully documented evidence of the minimally important difference (MID) of the scale. This was evaluated in a study that was designed to assess the magnitude of change in the LAEP and other PROs in order to exclude chance or error at various levels of certainty in patients with medically refractory epilepsy through application of a Reliable Change Index analytic approach [44]. Two PROs had partial evidence of MID; the MID investigation for the QOLIE-31 provided evidence for the total score but none of the sub-scales and for the QOLIE-89 results were provided for selected subscales and total score. No anchor-based values for interpreting change were reported for any of the reviewed instruments, and none reported responder definitions for an epilepsy population according to the FDA's requirements around establishing responder definitions (see Table 1). 
Table 3 Instrument review against regulatory requirements

\begin{tabular}{|c|c|c|c|c|c|c|c|c|}
\hline \multirow{2}{*}{$\begin{array}{l}\text { Instrument } \\
\text { abbreviated name }\end{array}$} & \multirow{2}{*}{$\begin{array}{l}\text { Conceptual } \\
\text { framework }\end{array}$} & \multicolumn{2}{|c|}{ Validity: content } & \multirow{2}{*}{$\begin{array}{l}\text { Validity: } \\
\text { construct }\end{array}$} & \multirow{2}{*}{$\begin{array}{l}\text { Reliability: } \\
\text { test-retest }\end{array}$} & \multirow{2}{*}{$\begin{array}{l}\text { Reliability: } \\
\text { internal } \\
\text { consistency }\end{array}$} & \multirow{2}{*}{$\begin{array}{l}\text { Ability } \\
\text { to } \\
\text { detect } \\
\text { change }\end{array}$} & \multirow{2}{*}{$\begin{array}{l}\text { Interpretation } \\
\text { of change }\end{array}$} \\
\hline & & $\begin{array}{l}\text { Patient } \\
\text { input }\end{array}$ & $\begin{array}{l}\text { Literature \& clinician } \\
\text { input }\end{array}$ & & & & & \\
\hline ABNAS & $x$ & Partial & $\checkmark$ & Partial & Partial & Partial & - & - \\
\hline $\begin{array}{l}\text { Attack Symptom } \\
\text { Measure }\end{array}$ & $x$ & $x$ & $\checkmark$ & Partial & - & Partial & - & - \\
\hline BPSE & $x$ & $x$ & $\checkmark$ & Partial & - & Partial & - & - \\
\hline EFA & $x$ & $\checkmark$ & $x$ & Partial & $\checkmark$ & Partial & - & - \\
\hline EPSES & $x$ & Partial & $\checkmark$ & Partial & Partial & - & - & - \\
\hline EPI-QOL & $x$ & Partial & $\checkmark$ & $\checkmark$ & Partial & Partial & - & - \\
\hline ESES & $x$ & Partial & Partial & Partial & Partial & $\checkmark$ & - & - \\
\hline ESI-55 & $x$ & Partial & $\checkmark$ & $\checkmark$ & - & Partial & $\checkmark$ & - \\
\hline $\mathrm{ICl}$ & $x$ & Partial & $\checkmark$ & $\checkmark$ & - & $\checkmark$ & - & - \\
\hline IES & $x$ & Partial & $\checkmark$ & $\checkmark$ & - & $x$ & - & - \\
\hline LAEP & $x$ & Partial & $x$ & Partial & $\checkmark$ & $\checkmark$ & - & $\checkmark$ \\
\hline LSSS & $x$ & $\checkmark$ & $\checkmark$ & Partial & Partial & Partial & Partial & - \\
\hline NDDI-E & $x$ & $x$ & $\checkmark$ & $\checkmark$ & $\checkmark$ & $\checkmark$ & - & - \\
\hline NEWQOL & $x$ & Partial & $\checkmark$ & Partial & Partial & Partial & - & - \\
\hline $\begin{array}{l}\text { Perceived Limitations } \\
\text { Scale }\end{array}$ & $x$ & $x$ & $x$ & - & - & $x$ & - & - \\
\hline Perceived Stigma Scale & $x$ & $x$ & $x$ & - & - & $x$ & - & - \\
\hline PESOS & $x$ & $x$ & $\checkmark$ & Partial & Partial & Partial & $\checkmark$ & - \\
\hline PNS & $x$ & $x$ & $\checkmark$ & Partial & $\checkmark$ & - & - & - \\
\hline QOLIE-10 & $x$ & Partial & $\checkmark$ & Partial & Partial & $x$ & Partial & - \\
\hline QOLIE-31 & $x$ & Partial & $\checkmark$ & Partial & Partial & Partial & $\checkmark$ & Partial \\
\hline QOLIE-89 & $x$ & Partial & $\checkmark$ & Partial & Partial & Partial & $\checkmark$ & Partial \\
\hline SEALS & $x$ & Partial & $\checkmark$ & $\checkmark$ & Partial & Partial & - & - \\
\hline SHE & $x$ & $\checkmark$ & $\checkmark$ & $\checkmark$ & $\checkmark$ & Partial & - & - \\
\hline SIDAED & $x$ & $x$ & $x$ & - & - & - & - & - \\
\hline SSQ & $x$ & Partial & $\checkmark$ & Partial & Partial & - & - & - \\
\hline WPSI & $x$ & $x$ & $\checkmark$ & Partial & Partial & Partial & Partial & - \\
\hline
\end{tabular}

\section{Discussion}

This review sought to evaluate epilepsy-specific PRO instruments against FDA regulatory standards and to identify gaps in the development process of the instruments that would need to be addressed prior to regulatory review. Twenty-six (26) epilepsy-specific PRO instruments were identified and reviewed.

This review identified that the SHE and NDDI-E met more of the regulatory requirements in terms of measurement properties, with both scales meeting four of the eight measurement properties evaluated. These two PROs lacked a published conceptual framework and require further evidence of ability to detect change and interpretation of change. In addition, the NDDI-E requires further evidence of patient input and the SHE requires further evidence of internal consistency reliability. Internal consistency fell below the required standard $(\alpha \geq 0.8)$ [85] for some of the SHE's sub-scales, further developmental work might be needed to increase the internal consistency of this scale (e.g. item removal, development of supplemental items, item re-wording, revised scale structure/conceptual framework). The SHE and NDDI-E were designed for different purposes. The SHE is a measure of patient's subjective handicap of epilepsy based on the World Health Organisation (WHO) concept of handicap, providing six subscale scores: work and activities; social and personal; physical; self-perception; life satisfaction; and change. The lack of focus on signs and symptoms in the SHE is likely to make it unfavourable from the FDA perspective as an instrument to support a PRO label claim. The same is true of other PROs evaluated in this review. The NDDI-E is a short instrument designed to detect depression in epilepsy patients, 
providing a total score. Whilst the NDDI-E has been used as an outcome measure in clinical trials [90,91], this may have been inappropriate as it was designed as a screening tool, and therefore its ability to detect change is not only unknown but potentially unlikely given that it was not designed to capture change.

The LAEP, ICI and ESI-55 all met requirements for three of the eight measurement properties. The LAEP in terms of test-retest reliability, internal consistency and interpretation of change; the ICI in terms of literature and clinician input, construct validity and internal consistency; and the ESI-55 in terms of literature and clinician input, construct validity and ability to detect change. Therefore all three instruments lack a published conceptual framework and required further evidence in terms of patient input, as well as specific gaps for each PRO.

When considering PROs with at least partial evidence of measurement properties, a different group of PROs come to the forefront: the QOLIE-31 and QOLIE-89 met or partially met requirements for seven out of the eight measurement properties, and the LSSS met or partially met requirements for six of the measurement properties. However, of concern for the QOLIE-89 is that seizure free patients did not score significantly higher than the most severe group on nine of the sub-scales [64] and test-retest reliability was $<0.7$ for four of the sub-scales [64]. Of concern for the QOLIE-31 is that internal consistency was $<0.8$ for four sub-scales [60] (although at 0.77-0.79 they were very close to this threshold) and test-retest fell below 0.7 for one sub-scale [60]. Questions are raised over the LSSS because of gaps in the evidence for reliability and validity.

Of particular concern from a regulatory perspective are the Perceived Limitations Scale, Perceived Stigma Scale and the SIDAED, all of which failed to achieve even partial evidence for any of the eight measurement characteristics. Notably, the Perceived Limitations Scale and Perceived Stigma Scale failed to achieve required standards for internal consistency when tested (no evidence of testing for this for the SIDAED). No other psychometric properties were tested for these three PROs.

Content validity is defined by the FDA as ' ... the extent to which the instrument measures the concept of interest', with evidence being supported through the conduct of qualitative studies to demonstrate that the items and domains of an instrument are appropriate and comprehensive relative to its intended measurement concept, population and use [5]. Importantly, whilst all the evidence criteria in this review are considered to be required by the FDA (with the exception of responder definitions which are recommended) the FDA make a clear statement that 'It is important to establish content validity before other measurement properties are evaluated'. The FDA will review the process for evidence of content validity in terms of item generation, data collection method and instrument administration mode, recall period, response options, instrument format, instructions and training, patient understanding, scoring of items and domains, and respondent and administration burden. The evaluation of content validity in this review has been an assessment of process rather than an evaluation of the evidence for content validity, with access restricted to published information rather than the detailed qualitative results that would be required for FDA assessment of content validity. No published conceptual framework was identified for any of the instruments; however for nearly all of the reviewed PROs there is sufficient information regarding how items group into domains/concepts to be able to develop a conceptual framework. Compared to other deficiencies, this may not be hard to overcome. Providing empirical evidence in support of the conceptual framework, would be a greater challenge.

In terms of evidence gathered through psychometric testing, the review identified common pitfalls. In relation to testing for construct validity, the FDA require that $a$ priori hypotheses are tested concerning logical relationships that should exist with measures of related concepts or scores. This was an area where most PROs failed to meet the criteria in this review. It is entirely possible that hypotheses were set in advance of analysis, but not reported in the published manuscript and it is strongly encouraged that this information is shared in publications reporting the psychometric properties of PROs. In terms of test-retest reliability and internal consistency, it is necessary to provide evidence for all domains and total scores (if applicable). Again this was a common area where PROs failed to meet the criteria in this review.

Although several PROs had evidence from clinical studies that the PRO measured change in an epilepsy population, few PROs had evidence that ability to detect change had been specifically investigated. One likely reason for this is that this measurement property is harder to evaluate in terms of study design as it means undertaking a longitudinal study of a patient group expected to improve/ deteriorate. However, this is an essential measurement property, particularly when considering that the PROs are to be incorporated into clinical studies designed to test the effectiveness of a treatment for epilepsy.

Even fewer PROs had evidence for interpreting change or empirically derived responder definitions. The responder definition may vary by target population or other clinical trial design characteristics and therefore the FDA will evaluate a PROs responder definition in the context of each clinical trial. Evidence towards a PROs MID can contribute to the responder definition, and so this review sought to identify any evidence of statistically derived or anchor-based MID and/or responder definition, and found that only the LAEP was able to demonstrate empirically derived evidence of the measurement property. 
One reoccurring problem found through the course of this review is that of several versions of one PRO instrument. Great care has to be taken when evaluating published evidence on the development and psychometric validation of a PRO instrument that the same version of the PRO is being referred to. By way of example, the ESES was originally developed to be a 25-item instrument, with evidence for content validity, construct validity and internal consistency [24]. However, the ESES was later updated to include an additional eight items to further assess self-efficacy associated with lifestyle issues with limited details around this update being published. In these instances, the evidence provided for earlier versions of the instrument were considered 'partial' evidence in Table 3 as this earlier evidence is likely to indicate how the revised instrument might perform. However, from a regulatory perspective the FDA would need to see full evidence for the developmental history and measurement properties of the revised instrument, assuming it is the revised version that is currently available for use in clinical trials.

It is vital to consider these findings in the broader context of PRO label claims in the US. The review of the current state of PRO label claims granted for new molecular entities approved 2006-2010 [18] found that of 116 products identified, $24 \%$ were granted PRO claims of which $86 \%$ were for symptoms and of these $38 \%$ were pain related. The proportion of new molecular entities and biologic license applications with PRO label claims has decreased slightly from 30\% 1997-2002 to 24\% 2006-2010. PRO label claims for non-primary endpoints were uncommon, with occurrence of symptoms the mostly commonly reported PRO label claim granted. The majority of accepted claims were supported by simple scales such as visual analogue scale (VAS), numeric rating scale (NRS), or symptom diaries, or on the basis of measures that have been traditionally accepted by the reviewing divisions. Within this context none of the reviewed epilepsy instruments are 'simple scales' i.e. VAS, NRS or symptom diaries. The reviewed PRO instruments do not appear to be measures that are 'traditionally accepted by the reviewing divisions' as none were identified in labels for PRO epilepsy treatments approved 2006-2010. The level of evidence required to support a desired label claim on the basis of the reviewed PRO instruments is of substantial importance as the reviewed instruments are not the typical PRO instruments being seen to support NME and BLA product approvals in the US.

It is worth considering the extent to which the evaluated PROs would be suitable to support regulatory approval of medicines in Europe through a regulatory review conducted by the EMA. The EMA has been less prescriptive in terms of their requirements for PROs, with one reflection paper published in 2005 for HRQL instruments, but nothing extended more generally to PROs. In the absence of clear guidelines from the EMA, it is difficult to determine which of the evaluated PROs would be well received by the EMA. However, whilst both the FDA and EMA require PROs supporting regulatory approvals to be validated and reliable, the EMA is more likely to accept and encourage the use of well-known, commonly used PROs than the FDA. The EMA also places less emphasis on qualitative evidence of content validity. Therefore PROs that are likely to be well received by the EMA will need to have demonstrated evidence of the psychometric properties of the PROs, particularly where this evidence is published in peer-review publication.

A limitation to this research is that the review was conducted on published information. It is frequently the case that documentation on the development process for PRO instruments is not published, particularly for older PRO instruments which were developed at a time where there were less publication options for PRO development manuscripts. It is likely that there are more details on the development of the reviewed instruments that have not been considered in this review. An important step for anyone considering the use of the PRO to support a label claim is to contact the instrument developer to see if further information can be made available to address seeming gaps in the evidence, which if available will reduce the need to conduct further research to gather evidence in support of the PRO instrument.

\section{Conclusions}

This systematic review of 26 epilepsy-specific PRO instruments, evaluated to the standards set out in the FDA guidance [5] indicates that none of the identified instruments have the full evidence required by the FDA to support the label claim, and all require further research to support their use as an endpoint. This may at least partially explain the lack of PRO label claims in support of epilepsy products. The SHE and NDDI-E have the fewest gaps that would need to be addressed through additional research prior to any FDA regulatory submission, although the NDDI-E was designed as a screening tool and is therefore unlikely to be suitable as an instrument for capturing change in a clinical trial and the SHE lacks the conceptual focus on signs and symptoms favoured by the FDA.

\section{Additional files}

Additional file 1: Description of data: Listing of search terms used through Embase and Medline.

\section{Abbreviations}

AEDs: Antiepileptic drugs; ABNAS: Aldenkamp-Baker neuroassessment Schedule; BPSE: Bonner psychsoziale skalen fur epilepsie; EFA: Epilepsy foundation of America; EMA: European Medicines Agency; EPSES: Epilepsy psycho-social effects scale; ESES: Epilepsy self-efficacy scale; ESI-55: Epilepsy 
surgery inventory 55; FDA: Food and Drug Administration; HRQL: Health related quality of life; ICl: Ictal consciousness inventory; IES: Impact of epilepsy scale; LAEP: Liverpool adverse events profile; LSSS: Liverpool seizure severity scale; MID: Minimally important difference; NDDI-E: The neurological disorders depression inventory for epilepsy; NEWQOL: Quality of life in newly diagnosed epilepsy; NRS: Numerical rating scale; PESOS: Performance, subjective evaluation and socio-demographic data; PNS: Portland neurotoxicity scale; PRO: Patient Reported Outcome; QOL: Quality of life; QOLIE: Quality of life in epilepsy; QOLIE-10: Quality of life in epilepsy 10; QOLIE-31: Quality of life in epilepsy 31; QOLIE-89: Quality of life in epilepsy 89; SEALS: Side effect and life satisfaction; SHE: Subjective handicap of epilepsy; SIDAED: Assessing side effects of AED treatment; SSQ: Seizure severity questionnaire; WHO: World Health Organisation; WPSI: Washington psychosocial seizure inventory.

\section{Competing interests}

The authors are employees of Oxford Outcomes, an ICON PIc. company. Oxford Outcomes received payment from Eisai for conducting the review and writing the manuscript. Eisai paid the article-processing charge.

\section{Authors' contributions}

AN: Study design, contributed to designing the search strategy, reviewed abstracts, developed the review criteria, reviewed the PRO instruments according to the criteria, led on manuscript development. CK: Study design, designed the search strategy, reviewed abstracts, developed the review criteria, reviewed the PRO instruments according to the criteria, contributed to manuscript development. KB: Contributed to designing the search strategy, reviewed abstracts, developed the review criteria, reviewed the PRO instruments according to the criteria, assisted with manuscript development. DW: Reviewed PRO instruments, reviewed the manuscript content. All authors read and approved the final manuscript.

\section{Acknowledgements}

The authors and Eisai would like to acknowledge the contribution of Dr Mallik Angalakuditi, formerly of Eisai, who originally commissioned the project from which this manuscript was developed, reviewed the literature search strategy, review criteria, final project report and the manuscript content.

Received: 1 August 2012 Accepted: 25 February 2013 Published: 11 March 2013

\section{References}

1. European Medicines Agency, Committee for Medicinal Products for Human Use: Guideline on clinical investigations of medicinal products in the treatment of epileptic disorders.. 22 ${ }^{\text {nd }}$ July 2010. http://www.ema.europa.eu/docs/ en_GB/document_library/Scientific_guideline/2010/01/WC500070043.pdf.

2. Commission on Classification and Terminology of the International League Against Epilepsy: Proposal for revised classification of epilepsies and epileptic syndromes. Epilepsia 1989, 30:389-399.

3. National Institute for Health and Clinical Excellence (NICE): The diagnosis and management of the epilepsies in adults and children in primary and secondary care. 2004. http://www.nice.org.uk/nicemedia/pdf/CG020fullguideline.pdf.

4. Schmidt D, Stavem K: Long-term seizure outcome of surgery versus no surgery for drug-resistant partial epilepsy: a review of controlled studies. Epilepsia 2009, 50(6):1301-1309.

5. Food and Drug Administration, U.S. Department of Health and Human Services: Guidance for Industry. Patient-Reported Outcome Measures: use in medicinal product development to support labeling claims. 2009. http://www. fda.gov/downloads/Drugs/GuidanceComplianceRegulatorylnformation/ Guidances/UCM193282.pdf.

6. Cramer JA: Quality of life for people with epilepsy. Neurol Clin 1994, 12:1-13.

7. Kerr C, Nixon A, Angalakuditi M: The impact of epilepsy on children and adult patient's lives: development of a conceptual model from qualitative literature. Seizure 2011, 20:764-774. http://dx.doi.org/10.1016/j. seizure.2011.07.007.

8. Baker GA, Marson AG: Cognitive and behavioural assessments in clinical trials: what type of measure? Epilepsy Res 2001, 45:163-167.

9. Leone MA, Beghi E, Righini C, Apolone G, Mosconi P: Epilepsy and quality of life in adults: a review of instruments. Epilepsy Res 2005, 66:23-44.
10. Stafford M, Gavriel S, Lloyd A: Patient-reported outcome measurements in epilepsy. Expert review in pharmacoeconomics. Outcomes Res 2007, 7:373-384.

11. Cramer JA: Assessing the severity of seizures and epilepsy: which scales are valid? Curr Opin Neurol 2001, 13:225-229.

12. Cramer JA: Principles of health-related quality of life: assessment in clinical trials. Epilepsia 2002, 43(9):1084-1095.

13. Cowan J, Baker GA: A review of subjective impact measures for use with children and adolescents with epilepsy. Qual Life Res 2004, 13:1435-1443.

14. Davis E, Waters E, Mackinnon A, Mackinnon A, Reddihough D, Graham H, Mehmet-Radji O, Boyd R: Paediatric quality of life instruments: a review of the impact of the conceptual framework on outcomes. Dev Med Child Neurol 2006, 48:311-318.

15. Waters E, Davis E, Ronen GM, Rosenbaum P, Livingston M, Saigal S: Quality of life instruments for children and adolescents with neurodisabilities: how to choose the appropriate instrument. Dev Med Child Neurol 2009, 51:660-669.

16. Selai CE, Trimble MR: Quality of life assessment in epilepsy: the state of the art. J Epilepsy 1995, 8:332-337.

17. Baker G, Smith D, Jacoby A, Hayes J, Chadwick D: Liverpool seizure severity scale revisited. Seizure 1998, 7:2001-2205.

18. Gnanasakthy A, Mordin M, Clark M, Demuro C, Fehnel S, Copley-Merriman C: A review of patient-reported outcome labels in the United States: 2006 to 2010. Value Health 2012, 15:437-442.

19. Elliot I, Lach L, Smith M: I just want to be normal: a qualitative study exploring how children and adolescents view the impact of intractable epilepsy on their quality of life. Epilepsy Behav 2005, 7:664-678.

20. Raty L, Soderfedlt B, Larrson B, Larrson G: Daily life in epilepsy: patients' experiences described by emotions. Epilepsy Behav 2007, 10:389-397.

21. Kilinc S, Campbell C: "It shouldn't be something that's evil, it should be talked about": a phenomenological approach to epilepsy and stigma. Seizure 2009, 18:665-671.

22. European Medicines Agency, Committee for Medicinal Products for Human Use: Reflection paper on the regulatory guidance for the use of health-related quality of life measures in the evaluation of medicinal products. 2006.

23. Revicki D, Hays RD, Cella D, Sloan J: Recommended methods for determining responsiveness and minimally important differences for patient-reported outcomes. J Clin Epidemiol 2008, 61:102-109.

24. Abetz L, Jacoby A, Baker GA, McNulty P: Patient-based assessments of quality of life in newly diagnosed epilepsy patients: validation of the NEWQOL. Epilepsia 2000, 41:1119-1128.

25. Aldenkamp A, Baker G, Pieters M, Schoemaker H, Cohen A, Schwabe S: The neurotoxicity scale: the validity of a patient based scale assessing neurotoxicity. Epilepsy Res 1995, 20:229-239.

26. Aldenkamp A, Baker G: The neurotoxicity scale II. Results of a patient based scale assessing neurotoxicity in patients with epilepsy. Epilepsy Res 1997, 27:165-173.

27. Aldenkamp A, Meel H, Baker G, Hendricks P: The A-B neuropsychological assessment schedule: ABNAS: the relationship between patientperceived drug related cognitive impairment and results of neuropsychological tests. Seizure 2002, 11:231-237.

28. Brooks J, Baker G, Aldenkamp A: The A-B neuropsychological assessment schedule: ABNAS: the further refinement of a patient based scale of patient-based scale of patient-perceived cognitive functioning. Epilepsy Res 2001, 43:227-237.

29. Goldstein L, Mellers J: Ictal symptoms of anxiety, avoidance behaviour and dissociation in patients with dossociative symptoms. J Neurol Neurosurg Psychiatry 2006, 77:616-621.

30. Helmstaedter C, Elger CE: Cognitive-behavioral aspects of quality of life in presurgical patients with epilepsy. J Epilepsy 1994, 7:220-231.

31. Gilliam F, Kuzniecky R, Faught E, Black L, Carpenter G, Schrodt R: Patientvalidated content of a epilepsy specific quality of life measurement. Epilepsia 1997, 38:233-236.

32. Gilliam F, Kuzniecky R, Meador K, Martin R, Sawie S, Viikinsalo M, Morawetz $R$, Faught E: Patient orientated outcome assessment after temporal lobectomy for refractory epilepsy. Neurology 1999, 53:687.

33. Loring DW, Larrabee GJ, Meador KJ, Lee GP: Dimensions of the epilepsy foundation concerns index. Epilepsy Behav 2005, 6:348-352.

34. Chaplin JE, Yepez R, Shorvon S, Floyd M: A quantitative approach to measuring the social effects of epilepsy. Neuroepidemiology 1990, 9:151-158. 
35. Piazzini A, Beghi E, Turner K, Ferraroni M: Health-related quality of life in epilepsy: findings obtained with a new Italian instrument. Epilepsy Behav 2008, 13:119-126.

36. Dilorio C, Schafer P, Letz R, Henry T, Schomer D, Yeager K, Project Ease Study Group: The association of stigma with self-management and perceptions of health care among adults with epilepsy. Epilepsy Behaviour 2003, 4:259-267.

37. Dilorio C, Faherty B, Manteuffel B: The development and testing of an instrument to measure self-efficacy in individuals with epilepsy. J Neurosci Nurs 1992, 24:9-13.

38. Langfitt J: Comparison of the psychometric characteristics of three quality of life measures in intractable epilepsy. Qual Life Res 1995, 4:101114

39. Vickrey BG, Hays RD, Graber J, Rausch R, Engel JJ, Brook RH: A healthrelated quality of life instrument for patients evaluated for epilepsy surgery. Med Care 1992, 30:299-319.

40. Cavanna AE, Mula M, Servo S, Strigaro G, Tota G, Barbagli D, Collimedaglia L, Viana M, Cantello R, Monaco F: Measuring the level and content of consciousness during epileptic seizures: the Ictal Consciousness Inventory. Epilepsy \& Behaviour 2008, 13:184-188.

41. Berg A, Langfitt J, Vickrey B, Wiebe S: Outcome Measures. In Epilepsy: A Comprehensive Textbook. 2nd edition. Philadelphia: Lippincott Williams \& Wilkins; 2007:1929-1937.

42. Jacoby A, Baker G, Smith D, Dewey M, Chadwick D: Measuring the impact of epilepsy: the development of a novel scale. Epilepsy Res 1993, 15:83-88.

43. Baker G, Jacoby A, Buck D, Stalgis C, Monnet D: Quality of life of people with epilepsy: a European study. Epilepsia 1997, 38:353-362.

44. Wiebe S, Eliasziw M, Matijevic S: Changes in quality of life in epilepsy: how large must they be to be real? Epilepsia 2001, 42:113-118.

45. Baker GA, Francis P, Middleton A, Jacoby A, Defalla B, Young C, Smith D, Chadwick DW: Development of a patient-based symptom checklist to quantify adverse events in persons receiving antiepileptic drugs. Epilepsia 1993, 34(Sup 6):18.

46. Baker G, Smith D, Dewey M, Jacoby A, Chadwick D: The initial development of a health-related quality of life model as an outcomes measure in epilepsy. Epilepsy Res 1993, 16:65-81.

47. Baker GA, Frances P, Middleton E, Jacoby A, Schaper GJ, Defalla B, Young C, Smith DF, Chadwick DW: Initial development, reliability, and validity of a patient-based adverse event scale. Epilepsia 1994, 35(suppl 7):80.

48. Baker GA, Jacoby A, Francis P, Chadwick DW: The Liverpool adverse drug events profile. Epilepsia 1995, 36:59

49. Perucca P, Carter J, Vahle V, Gilliam FG: Adverse antiepileptic drug effects. Neurology 2009, 72:1223-1229.

50. Baker G, Smith D, Dewey M, Morrow J, Crawford P, Chadwick D: The development of a seizure severity scale as an outcome measure in epilepsy. Epilepsy Res 1991, 8:245-251.

51. Scott-Lennox J, Bryant-Comstock L, Lennox R, Baker G: Reliability, validity and responsiveness of a revised scoring system for the Liverpool Seizure Severity Scale. Epilepsy Res 2001, 44:53-63.

52. Rapp S, Shumaker T, Smith T, Gibson P, Berzon R, Hoffman R: Adaptation and evaluation of the liverpool seizure severity scale and liverpool quality of life battery for American epilepsy patients. Qual Life Res 1998, 7:353-363.

53. Smith D, Baker GA, Jacoby A, Chadwick DW: The contribution of the measurement of seizure severity to quality of life research. Qual Life Res 1995, 4:143-158

54. Gilliam F, Barry JJ, Hermann BP, Meador KJ, Vahle V, Kanner AM: Rapid detection of major depression in epilepsy: a multicentre study. Lancet Neurol 2006, 5:339-405.

55. Ryan R, Kemper K, Emlen A: The stigma of epilepsy as a self-concept Epilepsia 1980, 21:433-444

56. May T, Pfäfflin M: Evaluating comprehensive care: Description of the PESOS and its psychometric properties. In Comprehensive care for people with epilepsy. Edited by Engel J Jr, Pedley TA. John Libbey and Co; 2001:319-340.

57. Salinsky M, Storzbach D: The Portland neurotoxicity scale. Validation of a brief self-report measure of antiepileptic drug related neurotoxicity. Assessment 2005, 12:107-117.

58. Cramer J, Perrine K, Devinsky O, Meador K: A brief questionnaire to screen for quality of life in epilepsy: the QOLIE-10. Epilepsi 1996, 36:557-582.
59. Cramer JA, Arrigo C, Van Hammée G, Bromfield EB: Comparison between the QOLIE-31 and derived QOLIE-10 in a clinical trial of levetiracetam. Epilepsy Res 2000, 41:29-38.

60. Cramer JA, Perrine K, Devinsky O, Bryant-Comstock L, Meador K, Hermann BP: Development and cross-cultural translation of a 31-item quality of life questionnaire (QOLIE-31). Epilepsia 1998, 39:81-88.

61. Birbeck GL, Kim S, Hays RD, Vickrey BG: Quality of life measures in epilepsy: how well can they detect change over time? Neurology 2000, 54:1822-1827.

62. Birbeck GL, Hays RD, Cui X, Vickrey BG: Seizure reduction and quality of life improvements in people with epilepsy. Epilepsia 2002, 43:535-538.

63. Wiebe S, Matijevic S, Eliasziw M, Derry PA: Clinically important change in quality of life in epilepsy. J Neurol Neurosurg Psychiatry 2002, 73:116-120.

64. Devinsky O, Vickery BG, Cramer J, Perrine K, Herrman B, Meador K, Hays D: Development of the quality of life epilepsy inventory. Epilepsia 1995, 36:1089-1104

65. Kim S, Hays RD, Birbeck GL, Vickrey BG: Responsiveness of the quality of life in epilepsy inventory (QOLIE-89) in an antiepileptic drug trial. Qual Life Res 2003, 12:147-155.

66. Leidy NK, Elixhauser A, Rentz AM, Beach R, Pellock J, Schachter S, Willian MK: Telephone validation of the quality of life in epilepsy inventory-89 (QOLIE-89). Epilepsia 1999, 40:97-106.

67. Scoring manual for the quality of life in epilepsy inventory-89 (QOLIE-89). http://www.rand.org/content/dam/rand/www/external/health/ surveys_tools/qolie/qolie89_scoring.pdf.

68. Brown SW, Tomlinson LL: Anticonvulsant side effects: self-report questionnaire for use in community survey. Br J Clin Pract 1982, 18 (symposium suppl):147-149.

69. Gillham R, Baker G, Thompson P, Birbeck K, McGuire A, Tomlinson L, Eckersley L, Silveira C, Brown S: Standardisation of a self-report questionnaire for use in evaluating cognitive, affective and behavioural side effects of anti-epileptic drug treatment. Epilepsy Res 1996, 24:47-55.

70. Gillham R, Bryant-Comstock L, Kane K: Validation of the side effect and life satisfaction (SEALS) inventory. Seizure 2000, 9:458-463.

71. Kane K, Lee J, Bryant-Comstock L, Gillham R: Assessing the psychometric characteristics of the Side Effects and Life Satisfaction Inventory (SEALS) in epilepsy: further validation from lamotrigine clinical trials. Epilepsia 1996, 37(Suppl 5):4.

72. O'Donoghue M, Duncan J, Sandar W: The Subjective Handicap of Epilepsy Scale. A new approach to measuring treatment outcome. Brain 1998, 121:317-343.

73. Carpay J, Aldenkamp A, Van Donselaar C: Complaints associated with the use of antiepileptic drugs: results from a community-based study. Seizure 2005, 14:198-206

74. Gilliam F, Fesler A, Baker G, Vahle V, Carter J, Attarian H: Systematic screening allows reduction of adverse antiepileptic drug events. A randomised trial. Neurology 2004, 62:23-27.

75. Uij SG, Uiterwaal CS, Aldenkamp AP, van Donselaar CA: Study to improve the treatment of epilepsy. Epilepsia 2003, 44:S8.

76. Uijl SG, Uiterwaal CS, Aldenkamp AP, Carpay JA, Doelman JC, Keizer K, Vecht CJ, de Krom MC, van Donselaar CA: A cross-sectional study of subjective complaints in patients with epilepsy who seem to be well-controlled with anti-epileptic drugs. Seizure 2006, 15:242-248.

77. Uij SG, Uiterwaal CS, Aldenkamp AP, Carpay JA, Doelman JC, Keizer K, Vecht CJ, de Krom MC, van Donselaar CA: Adjustment of treatment increases quality of life in patients with epilepsy: a randomized controlled pragmatic trial. Eur J Neurol 2009, 16:1173-1177.

78. Cramer J, Baker G, Jacoby A: Development of a new seizure severity questionnaire: initial reliability and validity testing. Epilepsy Res 2002, 48:187-197.

79. Dodrill C, Batzel L, Queisser H, Temkin N: An objective method for the assessment of psychological and social problems among epileptics. Epilepsia 1980, 21:123-135.

80. Chang $\mathrm{CH}$, Gehlert S: The Washington Psychosocial Seizure Inventory (WPSI): psychometric evaluation and future applications. Seizure 2003, 12:261-267.

81. Wiebe S, Rose K, Derry P, McLachlan R: Outcome assessment in epilepsy: comparative responsiveness of quality of life and psychosocial instruments. Epilepsia 1997, 38:430-438.

82. Jacoby A: Epilepsy and the quality of everyday life. Findings from a study of people with well-controlled epilepsy. Soc Sci Med 1992, 6:657-666. 
83. Jacoby A: Felt verses enacted stigma: a concept revisited. Soc Sci Med 1994, 38:269-274.

84. Jacoby A, Baker GA, Steen N, Potts P, Chadwick DW: The clinical course of epilepsy and its psychosocial correlates: findings from a UK community study. Epilepsia 1996, 37:148-161.

85. Cronbach $\sqcup$ : Coefficient alpha and the internal structure of tests. Psychometrika 1951, 16:297-334.

86. Nunnally J, Bernstein I: Psychometric theory. New York: McGraw-Hill; 1994.

87. Baker GA, Spector S, McGrath Y, Soteriou H: Impact of epilepsy in adolescence: a UK controlled study. Epilepsy Behav 2005, 6:556-562.

88. Cramer JA, Brandenburg N, Xu X: Differentiating anxiety and depression symptoms in patients with partial epilepsy. Epilepsy Behav 2005, 6:569-653.

89. Bautista RED, Glen ET: Seizure severity is associated with quality of life independent of seizure frequency. Epilepsy Behav 2009, 16:325-329.

90. Naritoku DK, Warnock CR, Messenheimer JA, Borgohain R, Evers S, Guekht $A B$, Karlov VA, Lee Bl, Ríos Pohl L: Lamotrigine extended-release as adjunctive therapy for partial seizures. Neurology 2007, 69:1610-1619.

91. Fakhoury TA, Miller JM, Hammer AE, Vuong A: Effects of lamotrigine on mood in older adults with epilepsy and co-morbid depressive symptoms: an open-label, multicentre, prospective study. Drugs Aging 2008, 25:955-962.

doi:10.1186/1477-7525-11-38

Cite this article as: Nixon et al:: Patient Reported Outcome (PRO)

assessment in epilepsy: a review of epilepsy-specific PROs according to the Food and Drug Administration (FDA) regulatory requirements. Health and Quality of Life Outcomes 2013 11:38.

\section{Submit your next manuscript to BioMed Central and take full advantage of:}

- Convenient online submission

- Thorough peer review

- No space constraints or color figure charges

- Immediate publication on acceptance

- Inclusion in PubMed, CAS, Scopus and Google Scholar

- Research which is freely available for redistribution 